\title{
A NUMERICAL STUDY: LIQUID SLOSHING DYNAMICS IN A TANK DUE TO UNCOUPLED SWAY, HEAVE AND ROLL SHIP MOTIONS
}

\author{
T. Nasar ${ }^{1}$, S.A.Sannasiraj ${ }^{2}$ and V. Sundar ${ }^{2}$
}

${ }^{1}$ Professor, Department of Civil Engineering, Toc H Institute of Science \& Technology, Arakkunnam, Ernakulam-682313, India, Email: drnasar.thuvan@gmail.com

${ }^{2}$ Professor, Department of Ocean Engineering, Indian Institute of Technology Madras, Chennai - 600036, India, Email: sasraj@iitm.ac.in, vsundar@iitm.ac.in,

\begin{abstract}
:
In order to explore the physics implicated with the sloshing phenomenon subjected to independent regular sway, heave and roll excitations of the liquid tank system, theoretical studies are carried out. Four liquid fill levels with static liquid depth, $h_{s}$, to the length, l of aspect ratio ( $\left.h_{s} l\right) 0.163,0.325$, 0.488 and 0.585 , are considered. The energy spectra of sloshing oscillation, their qualitative assessment and the harmonics present in the sloshing oscillation are studied. Frequency-Response amplitude has also been presented. The study reveals that sway excites a particular mode of sloshing (primary harmonic) by fulfilling the resonance conditions and also excites secondary modes. However, the roll motion excites the first mode of sloshing irrespective of the excitation frequencies. The heave motion excites the particular mode which is assumed as an initial perturbation.
\end{abstract}

Keywords: Regular wave, perturbation, first mode, primary resonance, parametric resonance, barge, pitching.

\section{NOMENCLATURE}

$B \quad$ beam size of rectangular barge $(\mathrm{m})$

$f_{\mathrm{w}} \quad$ wave excitation frequency $(\mathrm{Hz})$

$H_{i} \quad$ incident wave height (m)

$L \quad$ non-dimensional length parameter

$X \quad$ sway $(\mathrm{m})$

$Z \quad$ heave $(\mathrm{m})$

$\Delta \quad$ displacement of the vessel $(\mathrm{kN})$

$\Omega \quad$ non-dimensional sloshing frequency

$\varepsilon \quad$ non-dimensional surface perturbation

$\phi \quad$ roll angle (radians)

$\gamma_{n} \quad$ forcing factor in horizontal motion

\begin{tabular}{|c|c|}
\hline$\kappa_{v}$ & $\begin{array}{l}\text { non-dimensional forcing amplitude in } \\
\text { vertical motion }\end{array}$ \\
\hline$\rho$ & density of fluid in the tank $\left(\mathrm{kg} / \mathrm{m}^{3}\right)$ \\
\hline$\omega$ & excitation frequency $(\mathrm{rad} / \mathrm{sec})$ \\
\hline$\omega_{n}$ & $\begin{array}{l}\text { natural sloshing frequency }(\mathrm{rad} / \mathrm{sec}) \text {, } \\
\mathrm{i}=1,2,3 \ldots \ldots\end{array}$ \\
\hline & horizontal excitation frequency $(\mathrm{rad} / \mathrm{sec})$ \\
\hline & $\begin{array}{l}\text { vertical excitation frequency }(\mathrm{rad} / \mathrm{sec}) \\
\text { sloshing oscillation }(\mathrm{m})\end{array}$ \\
\hline
\end{tabular}

$\zeta \quad$ sloshing oscillation (m)

\section{Introduction}

The oscillation of liquid inside a partially filled container due to external disturbance is termed as 'sloshing'. The liquid sloshing in a LNG carrier, tanks of oil carrier, water flow on the decks of ship and their associated dynamic response are of great concern to hydro - dynamists and naval architects. With the ever increasing energy demand around the globe, the cargo capacities of LNG and FPSO tankers have increased from $145000 \mathrm{~m}^{3}$ to $241000 \mathrm{~m}^{3}$ within a decade (British Maritime corporate profile, 2005). Further, the International Energy Agency (IEA) estimates that by 2030, there will be a six fold increase in the number of LNG tanker ships in operation worldwide (The Naval Architect, 2007). It emphasizes on safety, particularly around the sloshing of LNG in heavy seas.

Fig. 1 shows the interaction problem and the traditional approach to deal it. For the simplicity of analysis, the sloshing induced by the vessel motion can be divided into sloshing problem and sea keeping problem. The liquid inside the tank responds to the vessel motion and in turn the motion of the liquid induces force. The sloshing forces transmitted to tank structure and floating vessel can be obtained from the integration of pressures on the tank walls. The motion of the vessel can then be solved for the combined sloshing and wave forces. 

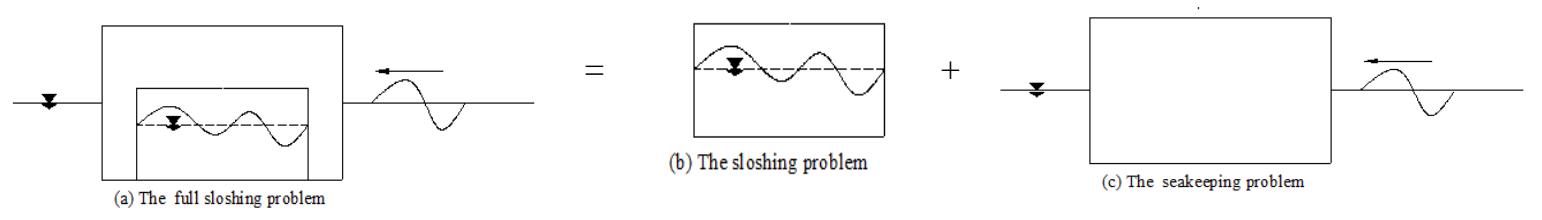

Fig. 1: The interaction problem of sloshing and the floating vessel.

In view of the importance of the liquid sloshing dynamics in the maritime industry, it is necessary to understand the physics of the sloshing phenomenon in a tank. The resonance sloshing would be severe if wave contains the frequency content of corresponding partial filled condition even if the ship does not experience extreme motion. Recent trends in the adoption of double-hulled structure and reduction of internal structural members especially in VLCC (Very Large Crude Carrier) pose intensification of sloshing load in cargo oil tanks and in ballast tanks. Hence, it is necessary to explore the possible liquid sloshing resonance conditions for which the sloshing pressure on the liquid containment would be severe. The intensity of the liquid sloshing pressure depends on the type of sloshing wave that occurs in the system. In general, the type of excitation i.e., translational (sway, surge and heave) / rotational (roll, pitch and yaw) motion of the tank decide the type of wave formation of the free surface of partially filled tank.

\section{Literature Review}

Considerable studies have been carried out to understand the complex sloshing phenomena through analytical, numerical and experimental methods. Benjamin and Ursell (1954) investigated the stability chart for the Faraday waves using linearized ideal fluid theory. The free surface oscillation in a vertically oscillating basin is called parametric sloshing or faraday wave, in which, the free surface movement is normal to the plane of excitation. Moiseyev (1958) has initiated the study on nonlinear resonant sloshing of liquid in a horizontally excited tank. A third order hydrodynamic potential theory for steady state sloshing induced by small amplitude sway/roll excitation was reported by Faltinsen (1974). Further, Faltinsen (1978) presented a nonlinear numerical model for predicting the sloshing in a rectangular tank subjected to forced harmonic oscillation in the sway mode.

In late nineties, considerable works have been devoted to predict sloshing surface elevation in the liquid tank subjected to translational/rotational excitation. A detailed review on the Faraday waves (sloshing waves in vertically oscillating tanks) has been reported by Miles and Henderson (1990). Jiang et al. (1996) carried out an experimental work and generated mild to steep Faraday waves in a narrow rectangular cylinder subjected to vertical oscillation. Instabilities in the frequency-sloshing amplitude (soft spring characteristics) have been explored. In addition, the modulation and temporal asymmetries in the sloshing oscillation were studied. Ockendon et al. (1986, 1993, and 1996) explored the existence of multiple periodic solutions and shock wave information present in the shallow water sloshing using analytical approach. Lui and Lou (1990) have carried out a linear analytical study to signify the importance of dynamic coupling between the liquid oscillation inside the tank and movement of the tank. Discrepancy in the roll response of the liquid - tank system was observed between liquid cargo and non shifting cargo while, the roll excitation frequency equals the sloshing frequency. Waterhouse (1994) elucidated the importance of critical depth $\left(h_{\mathrm{s}} / l\right)$ of 0.337 , which delineates hard spring and soft spring characteristics. Solass and Faltinsen (1997) developed a semi-analytical approach to study twodimensional (2-D) sloshing in the sway excited tanks. Extensive studies on modal analysis of liquid sloshing have been carried out (Faltinsen et al., 2000; Faltinsen and Timokha, 2001; Faltinsen and Timokha, 2002). The possible harmonics present in the system for the aspect ratio ranging between $h_{\mathrm{s}} / l$ of 0.1 and 1.0 were explored. In general, the modal theories were limited without considering the liquid impact on the top deck of the tank, wave breaking and excessive run- up on tank walls.

A few of numerical approaches which are pertaining to the present work are also herein reported. Armenio and La Rocca (1996) carried out numerical study based on the shallow water hypothesis and validated with experimental work for the roll excitation. The study explored the formation of standing wave, traveling wave, hydraulic jump and breaking wave under the influence of roll excitation frequency and amplitude for the various liquid filling levels. Frandsen (2003) developed a finite difference based numerical scheme and sigma transformation mapping technique to simulate sloshing motion in the vertically excited containers. The importance of wave steepness on the initial perturbation and the associated nonlinear behaviour in free surface elevation has been discussed. In addition, Frandsen (2004) reported the effect of wave excitation amplitude on sloshing wave profiles. Also, the study presents the effect of excitation frequencies on sloshing oscillation and 
its qualitative assessment, energy spectra of sloshing oscillation and possible resonance conditions, for sway and heave excitations individually. Sriram et al. (2006) developed a numerical wave tank based on Finite Element Method (FEM) and investigated the effect of excitation frequency in the horizontal and the vertical excitation, separately. In the above said study, the possible sloshing modes that can occur in the system and the sloshing energy spectra have been explored in detail. Jermie et al. (2012) simulated the sloshing in a rectangular tank subjected to roll excitation using FEM formulation and explored the harmonics in the energy spectra for two different roll amplitudes and various frequencies. The sloshing resonance phenomenon in a spring mounted rectangular tank which idealizes steel making converter system is investigated by Pirker et al. (2012).

With the above background, the present study aims at exploring the physics associated with the sloshing phenomena by studying the sloshing oscillation profiles, sloshing energy spectra, possible resonance conditions and assessing the qualitative beahaviour of sloshing oscillation. The above parameters are studied for the individual sway, heave and roll excitations. The present study was supportive in analyzing the sloshing oscillation in a rectangular tank which was equipped in a barge and the barge was subjected to coupled mode of excitations (sway, heave and roll) in beam sea conditions. The results are presented in authors' following research publications: Nasar et al. [2008a \& 2008b], Nasar et al. (2009), Nasar et al. (2010) and Nasar et al. (2012).

\section{Analysis of Sloshing Oscillation}

\subsection{General}

The free surface oscillation of liquid in a partially filled tank is a highly non-linear phenomenon even when subjected to regular wave excitation through translation or rotational tank motion. However, the linear approximation of the resonant liquid sloshing frequencies $\left(f_{n}\right.$ in $\left.H z\right)$ for the $n$th mode is given as [Ibrahim, 2005],

$$
f_{n}=\frac{1}{2 \pi} \sqrt{\frac{n \pi g}{l} \tanh \left(\frac{n \pi h_{s}}{l}\right)}, n=1,2,3 \ldots \ldots
$$

where, $g$ acceleration due to gravity, $h_{s}$ static liquid depth, $l$ length of the tank along excitation direction. In a lateral (sway or surge) or rotational (roll or pitch) excited tank, sloshing oscillation would be severe at the principal resonance condition $\left(f=f_{n}\right)$, whereas, in a heave excited tank, principal parametric resonance condition $\left(f=2 f_{n}\right)$ might lead to higher sloshing oscillation. Two parametric instabilities, the so-called, primary instabilities occur, while, the wave excitation frequency, $f$ is equal to $2 f_{n} / m(m=1,3,5 \ldots)$ and; the secondary instabilities occur while, the $f=2 f_{n} / m,(m=2,4,6 \ldots)$.

\subsection{Sway excitation}

The definition sketch for the sway excited tank is shown in Fig. 2. The equation of motion of sloshing fluid for the tank subjected to the base acceleration of $X^{\prime \prime}(t)=-a_{h} \omega_{h}^{2} \cos (\omega t)$ is given [Warnitchai and Pinkaew (1998), Frandsen (2004)] as follows,

$$
m_{n} q_{n}^{\prime \prime}(t)+m_{n} \omega_{n}^{2} q_{n}(t)=\gamma_{n} X^{\prime \prime}(t), n=1,2,3 \ldots .
$$

where,

$$
m_{n}=\frac{1}{2}\left[\frac{\rho b l^{2}}{n \pi \cdot \tanh \left(\frac{n \pi h_{s}}{l}\right)}\right] ; \quad \omega_{n}^{2}=\frac{n \pi g}{l}\left[\tanh \left(\frac{n \pi h_{s}}{l}\right)\right] ; \quad \gamma_{n}=\left[\frac{\rho b l^{2}(1-\cos (n \pi))}{(n \pi)^{2}}\right]
$$

$q_{n}(\mathrm{t})$ is the time evolution function, $m_{n}$ is the modal mass at $n$th mode, $b$ is the tank dimension along third axis, and $a_{h}$ is the sway amplitude. The above equation can be solved by a numerical integration technique. Herein, Wilson - $\theta$ method has been adopted. Based on the potential theory, the first order surface elevation $\zeta(x, t)$ can be given as follows:

$$
\zeta(x, t)=\sum_{n=1}^{\infty} q_{n}(t) \cos \left(\frac{n \pi x}{l}\right)
$$




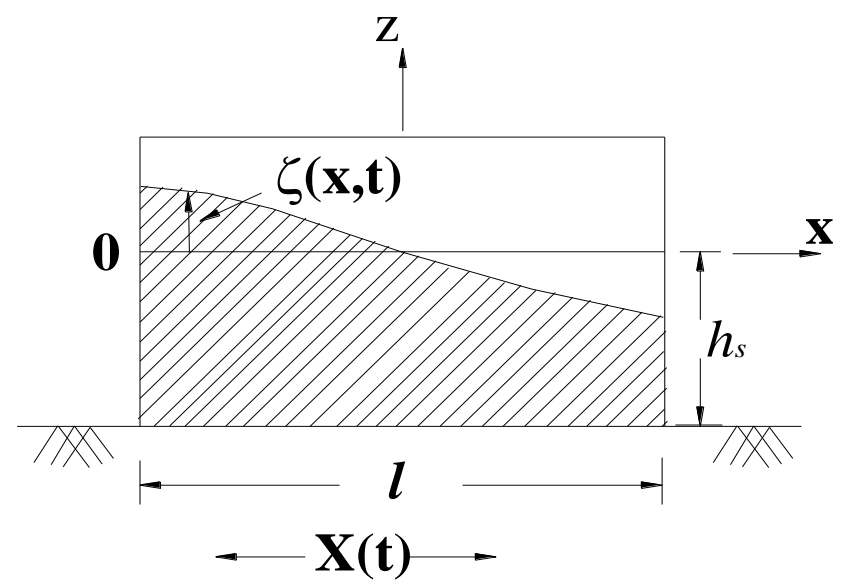

Fig. 2: Definition sketch of sloshing in a rectangular tank subjected to sway excitation.

The time histories of free surface elevation $\zeta(0, t)$ along the left wall of the tank, for an aspect ratio of $\left(h_{s} / l\right) 0.5$ subjected to base excitation frequency $\left(\omega_{h}\right)$ of $0.7 \omega_{1}$ is projected in Fig. 3. The present surface elevation result is compared with numerical result of Frandsen (2004) and agreement is found to be good. The surface elevation result is also compared (Fig. 4) with the numerical work results of Sriram et al. (2006).

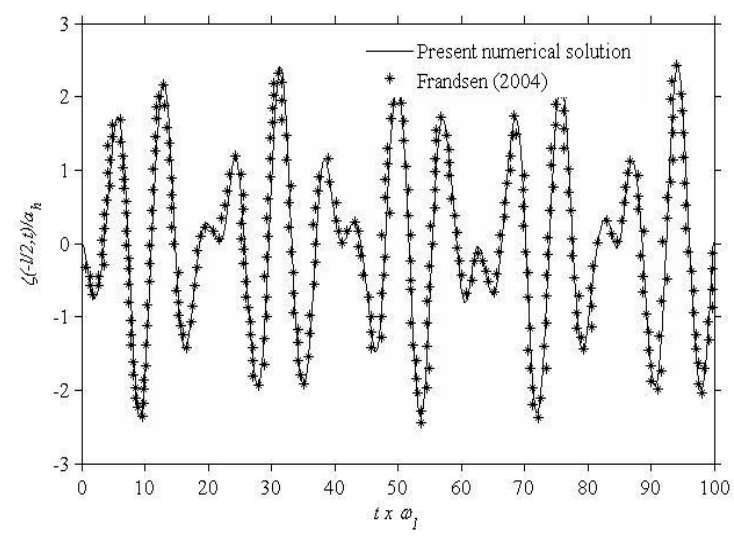

Fig. 3: Free surface elevation, $\zeta(-l / 2, t)$ at end of wall for an aspect ratio, $h_{s} / l=0.5$ subjected to sway excitation parameters: $a_{h}=0.005 h_{\mathrm{s}}$ and $\omega_{h}=$ $0.7 \omega_{1}$

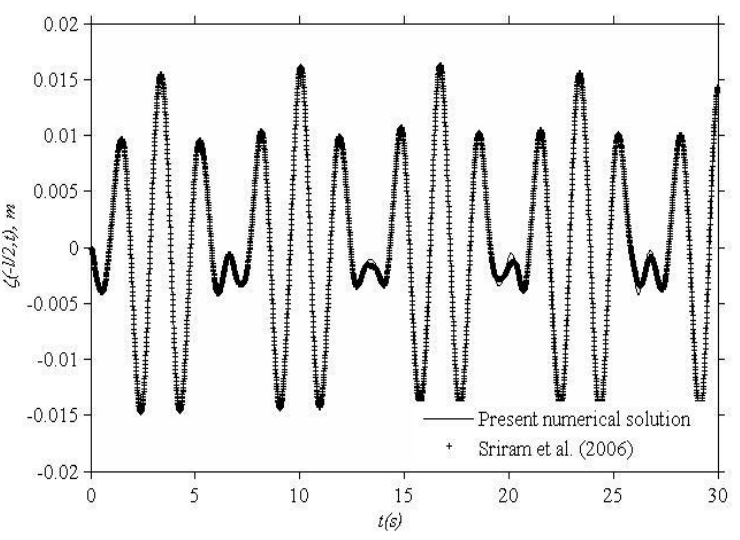

Fig. 4: Free surface elevation, $\zeta(-l / 2, t)$ at end of wall for an aspect ratio, $h_{s} / l=0.5$ subjected to sway excitation parameters: $a_{h}=0.005 h_{\mathrm{s}}$ and $\omega_{h}=$ $0.75 \omega_{1}$

The degree of nonlinearity in the surface elevation depends upon the number of modes associated in the system. Further, the surface wave modes and their superposition depend upon the fill level, tank geometry and wave excitation parameters (amplitude and frequency). Since, sloshing is a nonlinear phenomenon, the steady state amplitude - frequency response follows the nonlinear spring behaviour and its characteristics changes with fill level. The modal theory of Faltinsen et al. (2000) was exploited [Refer Appendix - A, Equation (A.5)] to study the amplitude - frequency response for fill levels of $\left(h_{s} / l\right) 0.163,0.325,0.488$ and 0.585 and are reported in Fig. 5. Sway excitation $(X)$ of $0.08 \mathrm{~m} /$ amplitude $\left(a_{h}\right)$ of $0.04 \mathrm{~m}$ was adopted for the present study. It is evident that the nonlinear amplitude - frequency response of an aspect ratio of 0.163 follows the hard spring characteristics, whereas, aspect ratio of 0.585 follows soft spring characteristics which are well away from critical water depth $\left(h_{\mathrm{s}} / l\right)$ of 0.337 . 

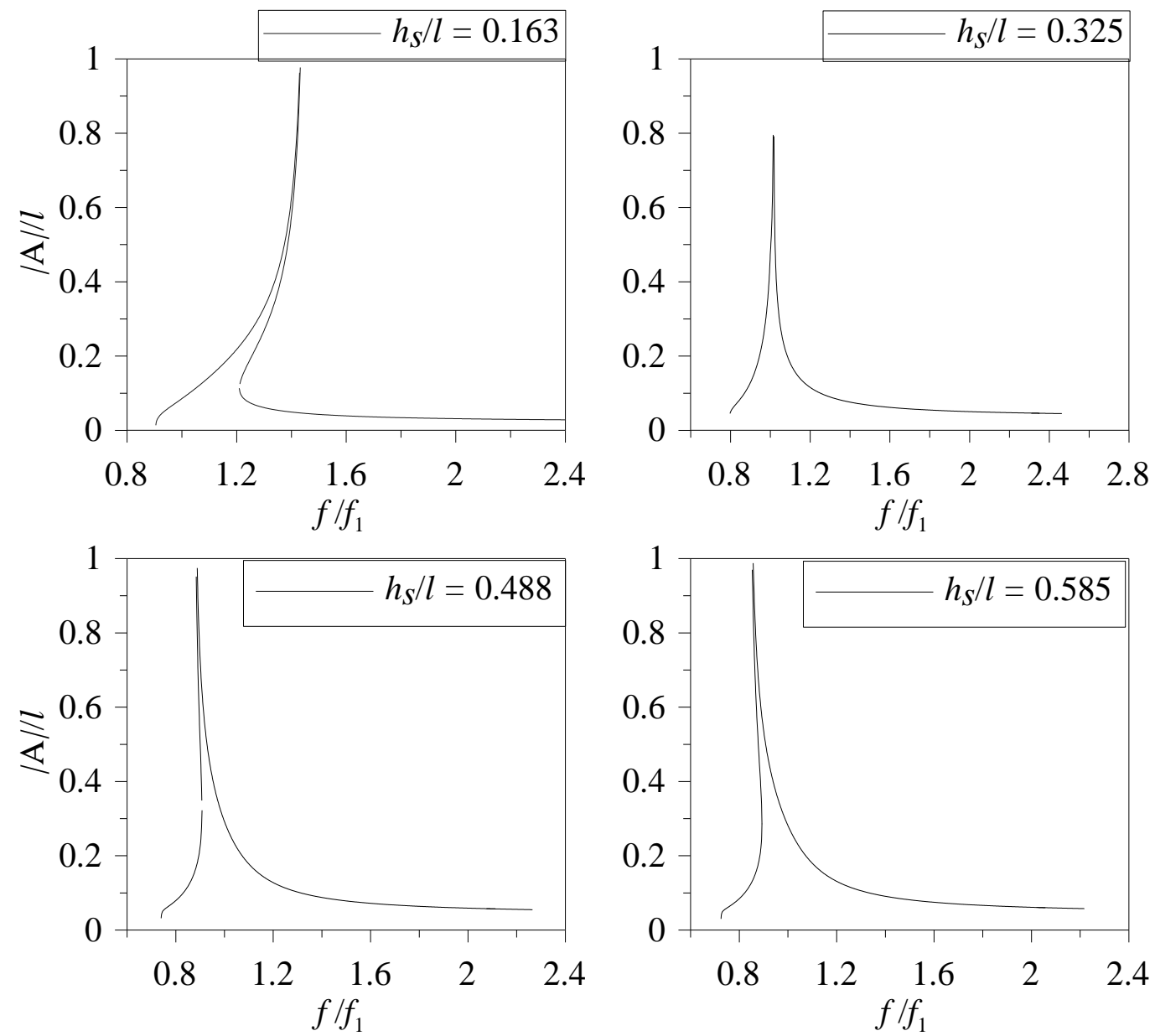

Fig. 5: Amplitude (A) - frequency response for different aspect ratio $\left(h_{s} / l\right)$ subjected to sway excitation $\left(a_{h}\right)$ of $0.04 \mathrm{~m}$.

Table 1: Tank sloshing frequencies for aspect ratio, $h_{s} / l=0.325$.

\begin{tabular}{cccccc} 
& $n=1$ & $N=2$ & $n=3$ & $n=4$ & $n=5$ \\
\hline$f_{n}(\mathrm{~Hz})$ & 0.78 & 1.23 & 1.54 & 1.78 & 1.99 \\
\hline$f_{n} / f_{1}$ & 1.0 & 1.61 & 1.96 & 2.28 & 2.55 \\
\hline $2 f_{n} / f_{1}$ & 2.0 & 3.22 & 3.92 & 4.56 & 5.10 \\
\hline
\end{tabular}

A parametric study was carried out to have insight knowledge about the modal behaviour of $\left(h_{s} / l\right) 0.325$. The normalized sloshing frequencies (Eqn.1) with $f_{1}$ up to fifth mode are provided in Table 1. However, the excitation frequency $(f)$ was considered between $0.46 \mathrm{~Hz}$ and $1.54 \mathrm{~Hz}$ (up to third mode) to comply with authors' experimental work of combined degree of excitation. The results of typical sloshing oscillation time histories and their corresponding phase plots subjected to excitation frequencies $(f)$ which are closer to the lowest mode sloshing frequencies $\left(f_{1}, f_{2}\right.$ and $\left.f_{3}\right)$ are depicted in Fig. 6. It can be seen that a growing in amplitude of sloshing was observed while the excitation frequency equals first mode $\left(f / f_{1}=1.0\right)$ and third mode sloshing frequency $\left(f l f_{1}=1.97\right)$. It is also learnt that the magnitude of sloshing is higher in first mode (Fig. 6a) than in third mode (Fig. 6c). Further, it is realized that odd mode sloshing frequencies give high response than even mode frequencies. An unstable behaviour is reflected in the phase plots (Fig. 6d and Fig. 6f) in response to increase in sloshing oscillation during its' time evolution. The sloshing oscillation at $f / f_{1}=1.61$ indicates the existence of multiple frequency components and the same is observed in the corresponding phase plot (Fig. 6e).

The steady state sloshing oscillation spectra for the various excitation frequencies are reported in Fig. 7. Results show that bimodal peaks (Fig. 7a and Fig. 7b) are observed at excitation frequency $(f)$ and at $f_{1}$ while the excitation frequency is lesser than first mode frequency $\left(f_{1}\right)$. A single energy peak (Fig. $\left.7 \mathrm{c}\right)$ can be seen while 
the $f$ equals the lowest mode sloshing frequency $\left(f_{1}\right)$ and also the energy value is high. Further, an increase in excitation frequency, $f=f_{2}$, the energy value starts diminish (Fig. 7d) at $f_{1}$ and concentrates at $f_{2}$ and $f_{3}$. However, the energy concentration is high at $f_{3}$ than at $f_{2}$. The results imply that the energy contribution by odd modes (likely to be excited by sway excitation) is higher than by even modes. A dominant energy peak is observed for the excitation at second lowest odd mode frequency, $f=f_{3}$ due to third mode contribution in the system (Fig. 7e). Similar kinds of trend in variation of spectral energy have been reported by Sriram et al. (2006).
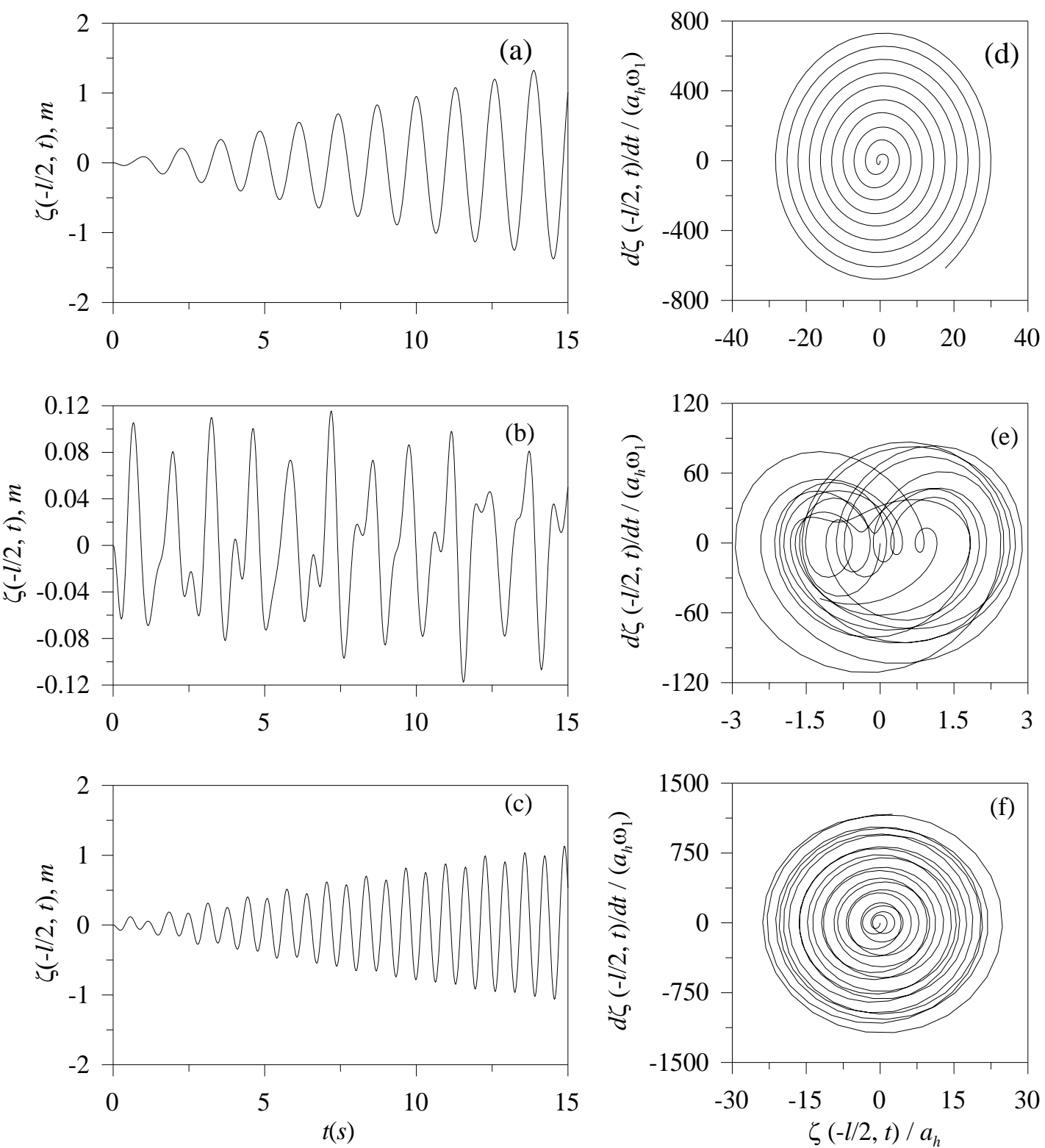

Fig. 6: Sloshing oscillation for $h_{s} / l=0.325$ subjected to sway excitation $X=0.08 \mathrm{~m}$ and: (a) $f / f_{1}=1.0$, (b) $f / f_{1}=$ 1.61, and, (c) $f / f_{1}=1.97$. The corresponding phase plots are (d), (e), and, (f).

Fig. 8 shows the definition sketch for the heave oscillation of the tank. An initial surface profile (perturbation) needs to be assumed for inducing sloshing oscillation in the liquid tank. In experimental work, initial perturbation is not possible, in the pure vertical mode of excitation. In the coupled mode of excitation, the initial disturbance can be either through sway or roll mode of excitations. However, for the present analytical study, an initial surface profile of $\zeta(x, t)=a \cos \left(k_{n} x\right)$ was assumed. The second order correction for the free surface elevation in a vertically base excited tank is given as Frandsen (2004): 
$\zeta(x, t)=a \cos \left(k_{n} x\right) Z_{n}{ }^{(1)}\left(\omega_{\mathrm{v}} t\right)+a\left(\frac{a \omega_{\mathrm{v}}^{2}}{\mathrm{~g}}\right) \cos \left(k_{2 n} x\right) Z_{n}{ }^{(2)}\left(\omega_{\mathrm{v}} t\right)$

where, $a$ is the initial perturbation of surface elevation in the tank, $\varepsilon=a \omega_{\mathrm{n}}^{2} / g$ and wave number, $k_{n}=n \pi / l$. The tank is assumed to be periodically excited with the base acceleration of $Z_{T}^{\prime \prime}=-\omega_{\mathrm{v}}^{2} a_{\mathrm{v}} \cos \left(\omega_{\mathrm{v}} t\right)$.
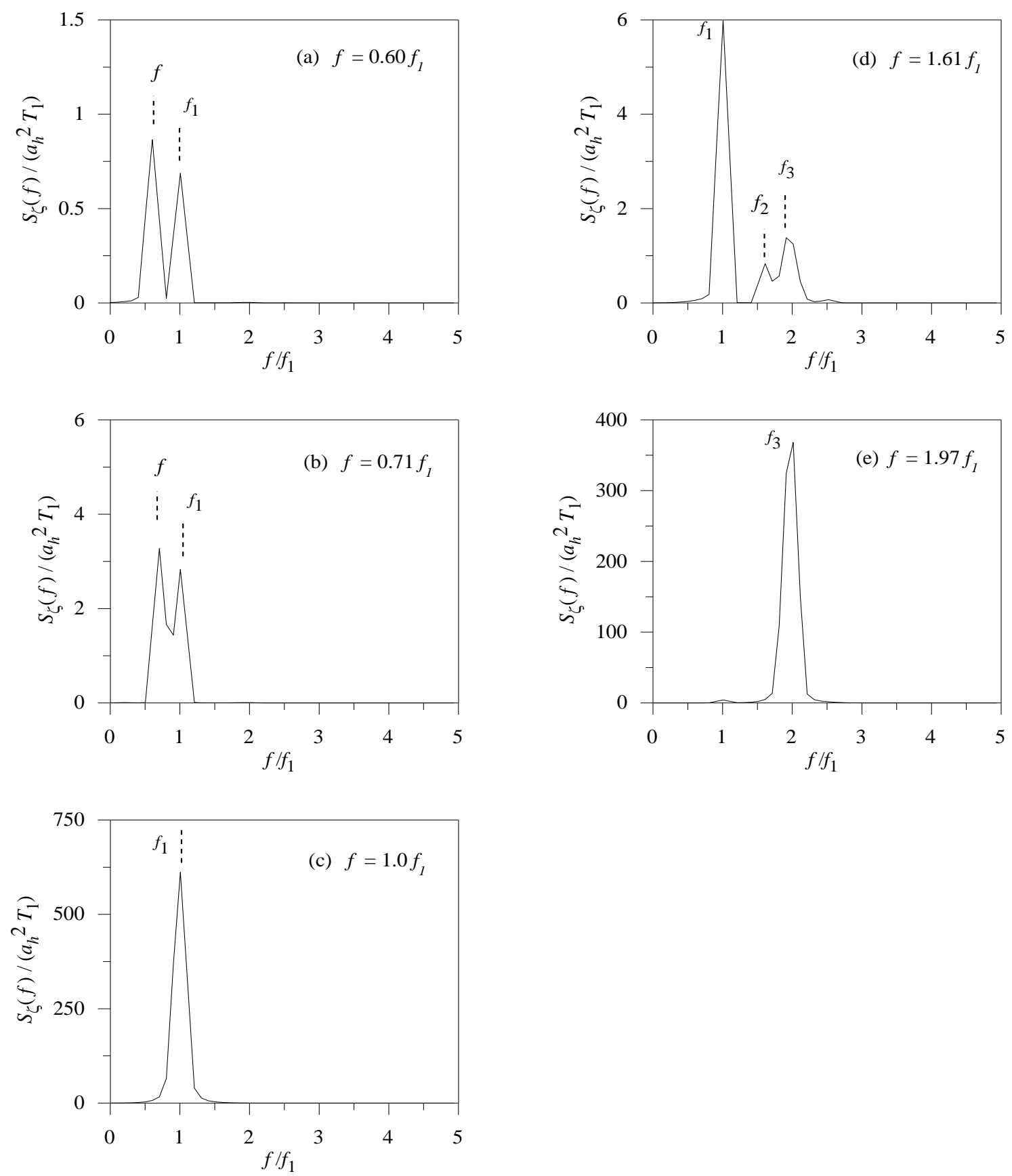

Fig. 7: Spectra of sloshing oscillation for $h_{s} l l=0.325$ subjected to various excitation frequencies with sway amplitude $\left(a_{h}\right)$ of $0.04 \mathrm{~m}$.

\subsection{Heave Excitation}

The evolution functions $Z_{n, 2 n}{ }^{(1,2)}$ should satisfy the homogeneous and non homogeneous Mathieu equations and it is given in nondimensional form as follows: 


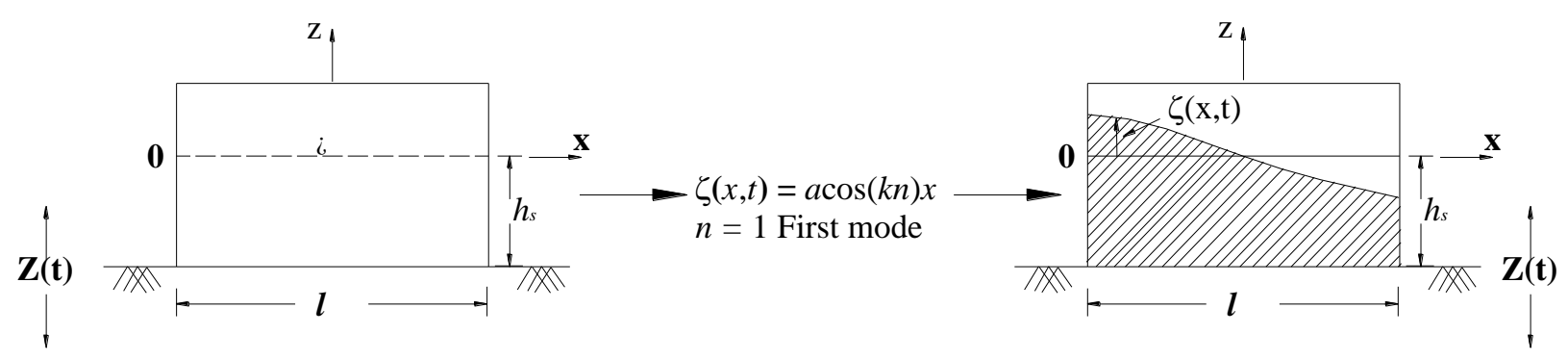

Fig. 8: Definition sketch of sloshing in a rectangular tank subjected to vertical excitation

$$
\begin{aligned}
& Z_{n}^{(1) "}\left(\omega_{\mathrm{v}} t\right)+\Omega_{n}^{2}\left(1+\kappa_{\mathrm{v}} Z^{\prime \prime}\left(\omega_{\mathrm{v}} t\right)\right) Z_{n}^{(1)}\left(\omega_{\mathrm{v}} t\right)=0 \\
& Z_{2 n}^{(2) "}\left(\omega_{\mathrm{v}} t\right)+\Omega_{2 n}^{2}\left(1+\kappa_{\mathrm{v}} Z^{\prime \prime}\left(\omega_{\mathrm{v}} t\right)\right) Z_{2 n}^{(2)}\left(\omega_{\mathrm{v}} t\right)=\frac{1}{2 \Omega_{n}^{2}}\left(\left(\frac{1}{2} \frac{\Omega_{2 n}^{2}}{\Omega_{n}^{2}}+2\right) \frac{k_{n}^{2}}{L^{2}}-\frac{\Omega_{2 n}^{2} \Omega_{n}^{2}}{2}\right) Z_{n}^{(1)^{\prime}}\left(\omega_{\mathrm{v}} t\right)^{2} \\
& -\left(1+\kappa_{\mathrm{v}} Z^{\prime \prime}\left(\omega_{\mathrm{v}} t\right)\right)\left(\frac{k_{n}^{2}}{L^{2}}-\frac{\Omega_{2 n}^{2} \Omega_{n}^{2}}{2}\right) Z_{n}^{(1)}\left(\omega_{\mathrm{v}} t\right)^{2}
\end{aligned}
$$

where, $\Omega_{n}=\frac{\omega_{n}}{\omega_{\mathrm{v}}}, \kappa_{\mathrm{v}}=a_{\mathrm{v}} \omega_{\mathrm{v}}^{2} / g, L=l \omega_{\mathrm{v}}^{2} / g, a_{v}$ is the heave amplitude and with the following nondimensional initial conditions, $Z_{n}{ }^{(1)}(\mathrm{O})=1, Z_{n}{ }^{(1)^{\prime}}(\mathrm{O})=\mathrm{O}, Z_{2 n}{ }^{(2)}(\mathrm{O})=\mathrm{O}$ and $Z_{2 n}{ }^{\left({ }^{(1)}\right.}(\mathrm{O})=\mathrm{O}$. Runge-Kutta fifth order time iteration method has been adopted to solve the non homogeneous Mathieu equations. The time evolution of sloshing oscillation subjected to excitation frequency $\left(\omega_{\mathrm{v}}\right)$ of $0.8 \omega_{1}$, heave amplitude $\left(a_{\mathrm{v}}\right)$ of $0.5 h_{s}$ and for $h_{\mathrm{s}} / l$ of 0.5 is projected in Fig. 9. An initial profile of first mode and its wave steepness $(\varepsilon)$ of 0.0014 was assumed. The result is found to be in accord with Frandsen (2004). The result (Fig. 10) is also compared with Sriram et al. (2006) for the wave excitation frequency $\left(\omega_{\mathrm{v}}\right)$ of $0.75 \omega_{1}$, wave steepness $(\varepsilon)$ of 0.014 and heave amplitude $\left(a_{\mathrm{v}}\right)$ of $0.005 h_{s}$.

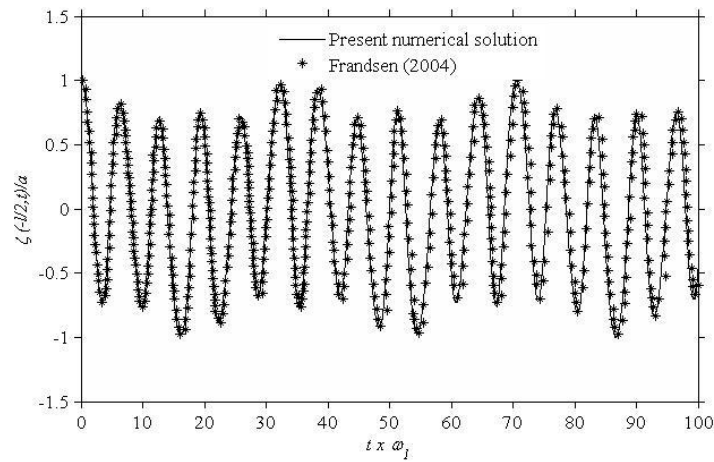

Fig. 9: Sloshing oscillation for $h_{s} l l=0.5$ due to heave excitation parameters, $\Omega_{1}=1.253, \kappa_{\mathrm{v}}=0.5$, $\varepsilon=0.0014$

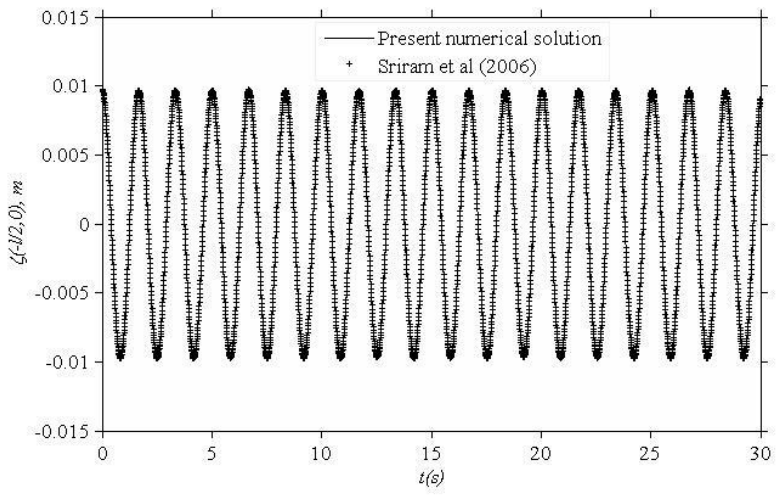

Fig. 10: Sloshing oscillation for $h_{s} / l=0.5$ due to heave excitation parameters, $\Omega_{1}=1.333$, $\kappa_{\mathrm{v}}=0.004, \varepsilon=0.014$, and with $h_{s} / l=0.5$

A parametric study has been carried out to explore the effect of initial surface assumption on spectral energy for an aspect ratio $\left(h_{\mathrm{s}} / l\right)$ of 0.325 . The normalized sloshing frequencies up to fifth mode and their corresponding parametric resonance condition for each mode are shown in Table 1. An initial perturbation parameter of $(\varepsilon)$ of 0.014 was considered of all the modes assumed.

The spectra of sloshing oscillation subjected to various excitation frequencies and for the assumption of first mode are shown in Fig. 11. In general, it can be seen that the sloshing energy is observed to be concentrate at first mode $\left(f / f_{1}=1\right)$ of all the frequencies considered. The variation of energy is not significant between $f=$ $0.65 f_{1}$ (Fig. 11a) and $f=1.61 f_{1}$ (Fig. 11e). However, the energy increases drastically while the excitation frequency $(f)$ approaches to the principal parametric resonance condition $\left(f=2 f_{1}\right)$. A bimodal peak has been 
observed (Fig. 11f) for the excitation frequency, $f=1.97 f_{1}$. A dominant peak at $f / f_{1}=1$ and secondary peak at excitation harmonics $\left(f f f_{1}=1.97\right)$ were identified due to the contribution of first of mode.
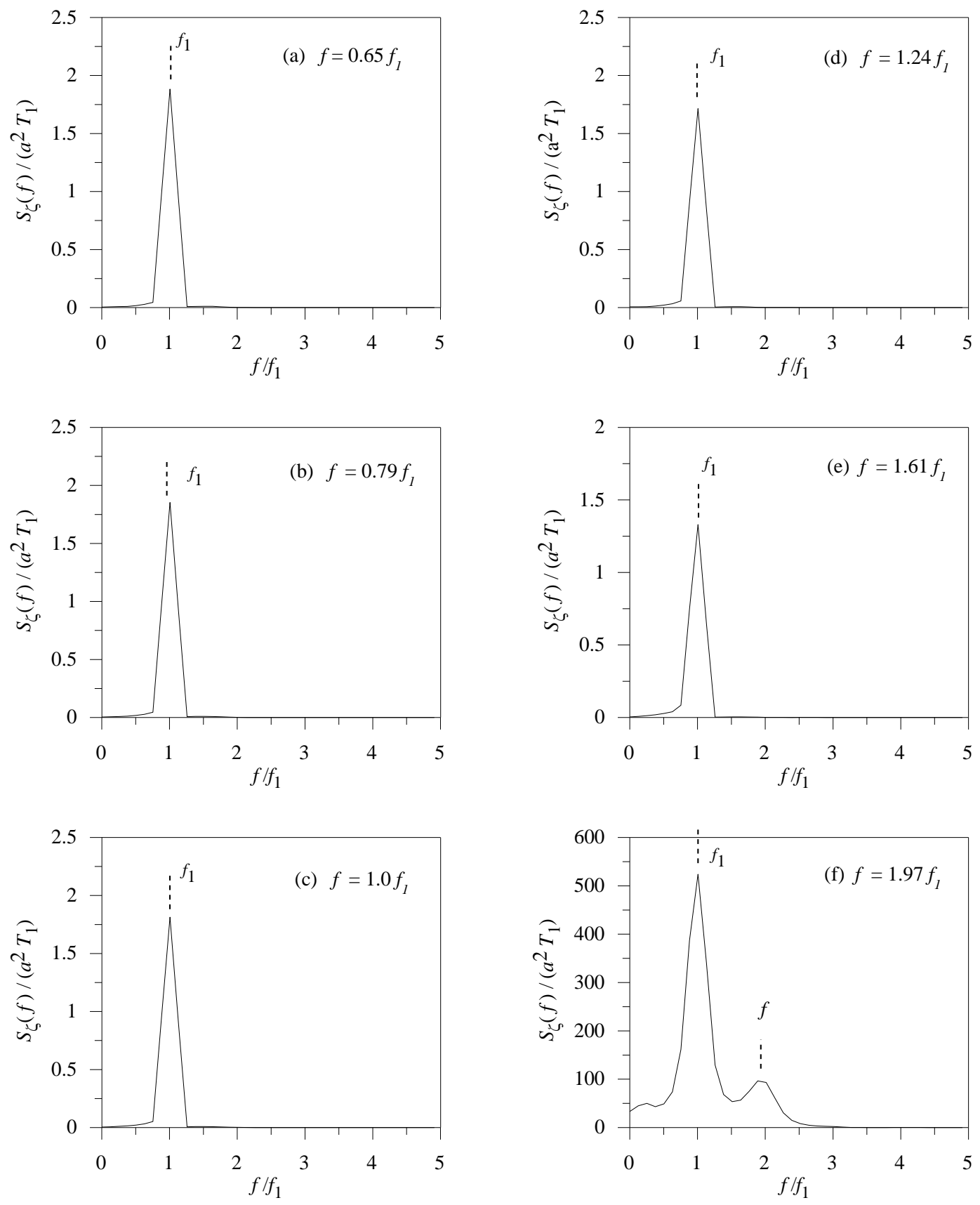

Fig. 11: Spectra of sloshing oscillation for $h_{s} l l=0.325$ subjected to various excitation frequencies with assumption of first mode $\zeta(x, t)=a \cos \left(k_{1} x\right)$.

Fig. 12 shows the spectral energy for the assumption of second mode as the initial profile in the heave excited tank. It is observed that energy is leaning to accumulate at $f / f_{1}=1.61$ (second mode, $f_{2} / f_{1}=1.61$ ) of all the excitation frequencies $(f)$ considered. Further, the energy increases as the excitation frequency tends towards the parametric resonance of second mode $\left(2 f_{2} / f_{1}=3.22\right)$. A similar kind of bimodal peak as shown in Fig. $12 \mathrm{f}$ has been observed as in the case of first mode assumption, for the excitation at $f / f_{1}=2.65$. The trend in the variation of spectral energy concentration for the third mode profile $\left(f_{3}\right)$ as shown in Fig. 13 is observed to be same as the lowest modes assumed $\left(f_{1}\right.$ and $\left.f_{2}\right)$. The energy concentrates at $f_{3} / f_{1}=1.96$. By analyzing the variation of spectral 
energy, it is inferred that the vertical mode of excitation increases the amplitude of sloshing oscillation of particular mode shape assumed as perturbation.
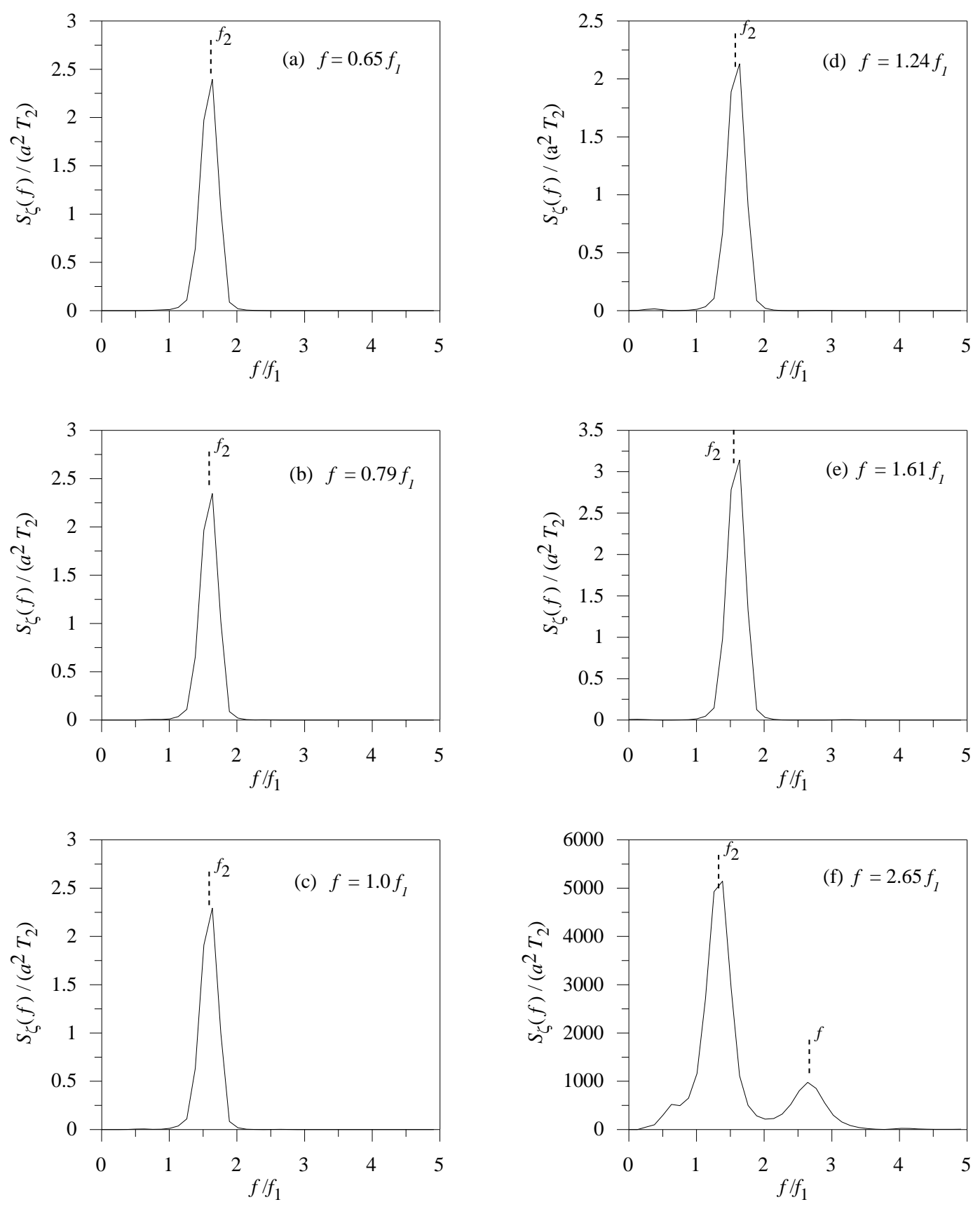

Fig. 12: Spectra of sloshing oscillation for $h_{s} / l=0.325$ subjected to various excitation frequencies with assumption of second mode, $\zeta(x, t)=a \cos \left(k_{2} x\right)$.

\subsection{Roll Excitation}

The definition sketch for the tank is being subjected to roll excitation is presented in Fig. 14. Let us assume that the tank is roll around the point $(0,0,-\mathrm{z})$ of mobile coordinate system with displacement of $\phi=\phi_{0} \sin (\omega t)$. The steady state sloshing amplitude in a rectangular tank excited by sinusoidal roll motion [(Faltinsen et al. (2000)] can be determined by the following Equation (7). 
$\left(r_{1}^{2}-1\right) \bar{A}+v\left(r_{2}, \bar{h}\right) \bar{A}^{3}-\bar{P}_{1} \phi_{o}\left(\frac{z_{1}}{l}-\frac{S_{1}}{l}+\frac{g}{l \omega^{2}}\right)=0$

where $\phi_{\mathrm{o}}$ is the roll amplitude, $z_{l}$ is the position of roll axis, $S_{n}=\frac{2 l}{n \pi} \tanh \left(\frac{n \pi}{2 l} h_{s}\right), n \geq 1$. The results for the aspect ratios of $0.163,0.325,0.488$ and 0.585 are depicted in Fig. 15. It is evident that $h_{\mathrm{s}} / l$ of 0.325 delineates the hard spring and soft spring characteristics.
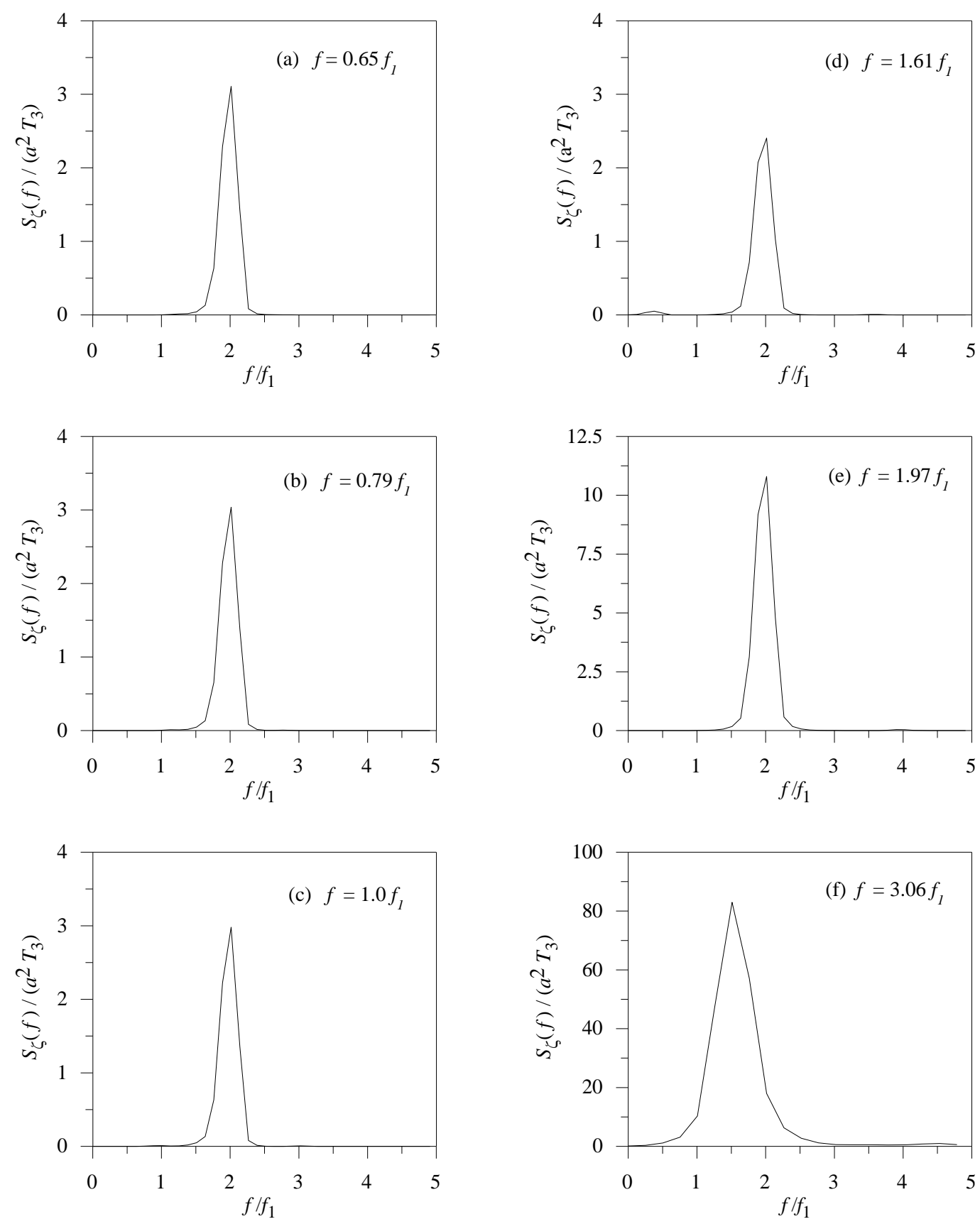

Fig. 13: Spectra of sloshing oscillation for $h_{s} / l=0.325$ subjected to various excitation frequencies with assumption of third mode $\zeta(x, t)=a \cos \left(k_{3} x\right)$. 


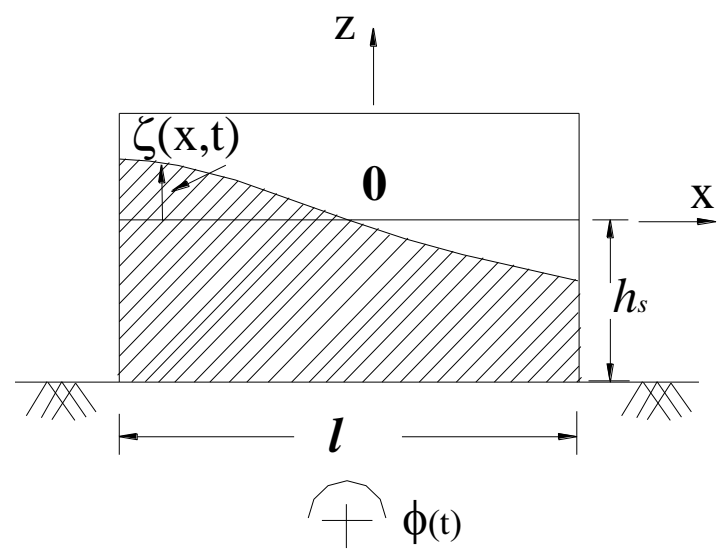

Fig. 14: Definition sketch of sloshing in a rectangular tank subjected to roll excitation
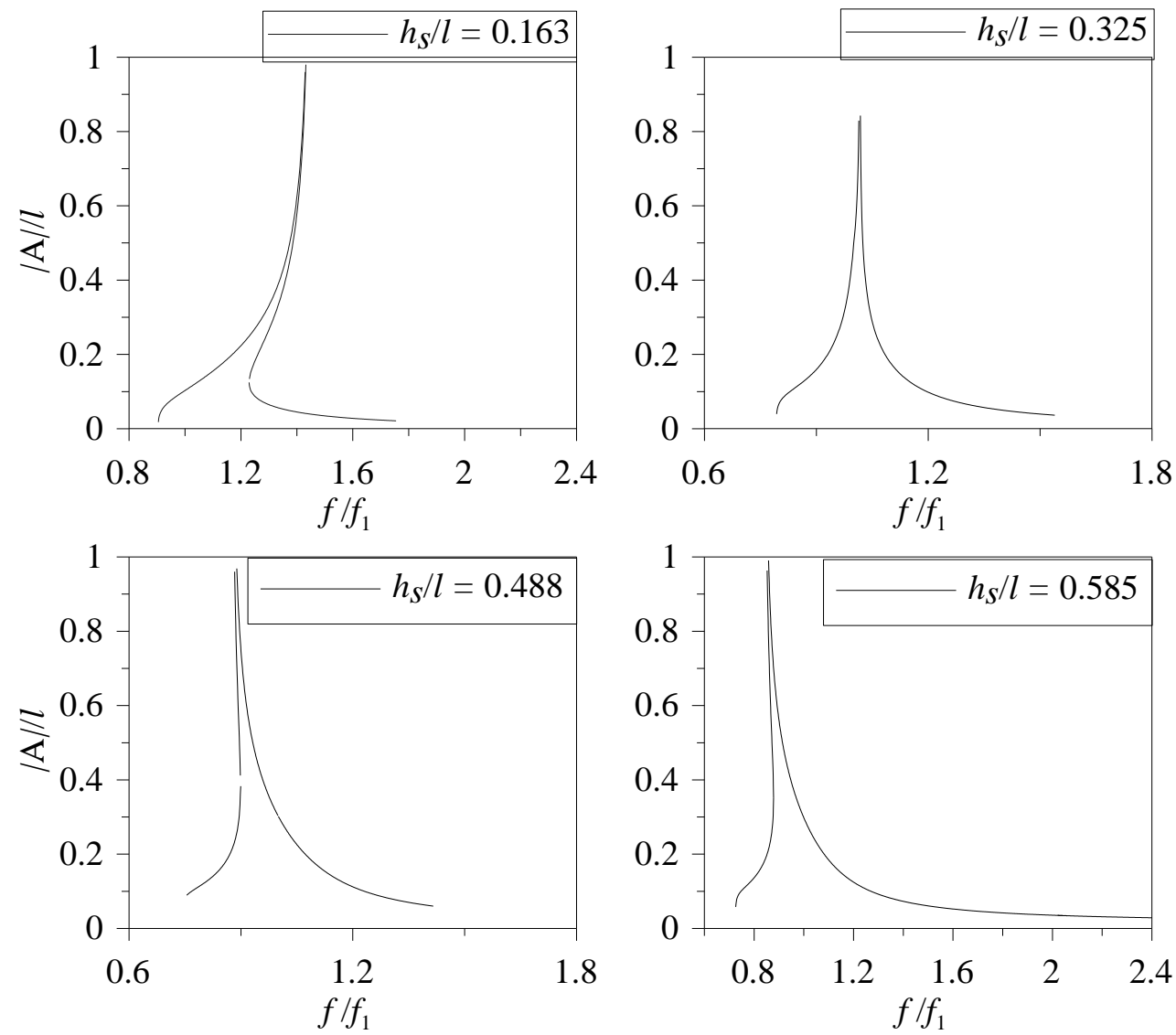

Fig. 15: Amplitude (A) - frequency response for different aspect ratio $\left(h_{s} / l\right)$ subjected to roll amplitude $\left(\phi_{\mathrm{o}}\right)$ of 0.1 radians

The free surface elevation (Faltinsen, 1974) inside the tank is given as follows:

$$
\zeta(x, t)=\zeta_{1} \phi_{o}^{1 / 3}+\zeta_{2} \phi_{o}^{2 / 3}+\zeta_{3} \phi_{o}
$$

where $\zeta_{1}, \zeta_{2}$ and $\zeta_{3}$ are the first-, second-, and third- order wave elevation respectively and are explained in Appendix $-\mathrm{B}$. The spectra of sloshing oscillation for different excitation frequencies and roll amplitude $\left(\phi_{0}\right)$ of 0.1 radians are shown in Fig. 16. It can be seen that the sloshing energy is observed to be concentrate on corresponding excitation frequencies and the energy is high at principal resonance condition $\left(f=f_{1}\right)$ (Fig. 16c). It 
is also inferred that sloshing energy concentrates (Fig. 16a-f) on first mode irrespective of the excitation frequencies considered and the same observation was reported by Jermie et al. (2012). In order to substantiate the above statement, the snap shot of sloshing profile inside the tank at various instant of time is given in Fig. 17. It is confirmed that the first mode alone induced in the liquid tank, even, if the excitation is at second mode $\left(f=1.61 f_{1}\right)$ (Fig. 17e) and third mode $\left(f=1.97 f_{1}\right)$ (Fig. 17f) sloshing frequencies.
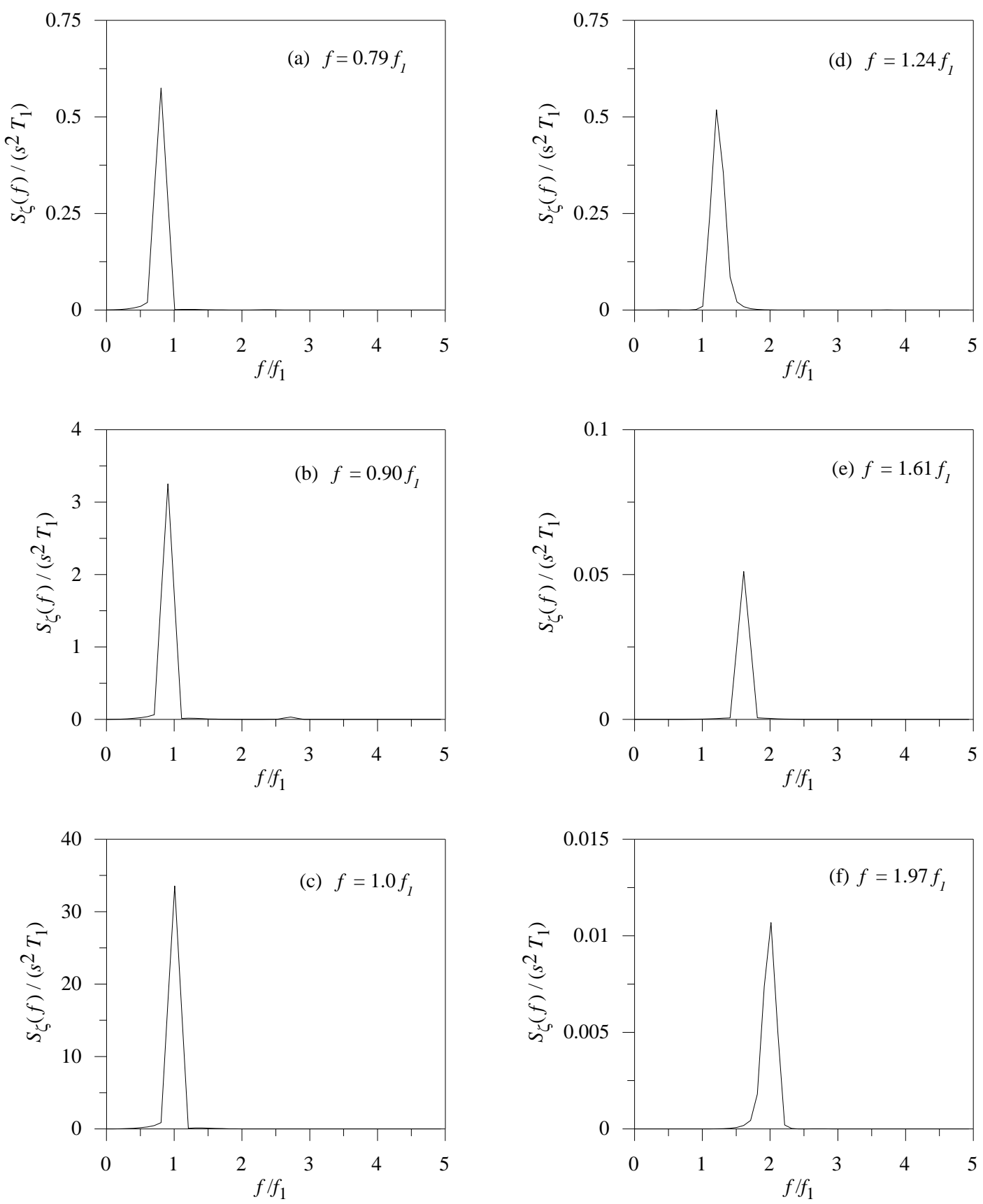

Fig. 16: Spectra of sloshing oscillation for $h_{s} / l=0.325$ subjected to various excitation frequencies with roll amplitude of $\phi_{\mathrm{o}}=0.1$ radians $\left(s=l / 2 * \phi_{\mathrm{o}}\right)$.

\subsection{Experimental Work (Coupled Sway, Heave and Roll Excitation)}

With the wide knowledge obtained from the individual degree of excitation (sway, heave and roll) and along with the inherent characteristics of a nonlinear system, the sloshing dynamics due to coupled degree of excitation was explored out. An excerpt from the authors' work Nasar et al. (2008a) is presented here. The time 
histories, the respective phase plots and the spectral density for the wave excitation frequency $\left(f_{w}\right)$ of $0.62 \mathrm{~Hz}$ (half of the second mode frequency) and due to barge excitation parameters $\left(X / H_{i}=0.70, Z / H_{i}=0.80\right.$ and $\phi B / 2 H_{i}=1.01$ ) are shown in Fig. 18. A bifurcation effect is observed in the phase plot (Fig. 18b) due to two equally dominant energy peaks as can be seen in Fig. 18c, one at $f_{w}$ and other at second mode frequency $\left(f_{2}\right)$. The sloshing at $f_{2}$ is due to the secondary parametric resonance of second mode $\left(f_{w} \cong f_{2} / 2\right)$. Also the surface profile as shown in Fig. 19 confirms the existence of the second mode and first mode (due to $f_{w}$ ) in the system.
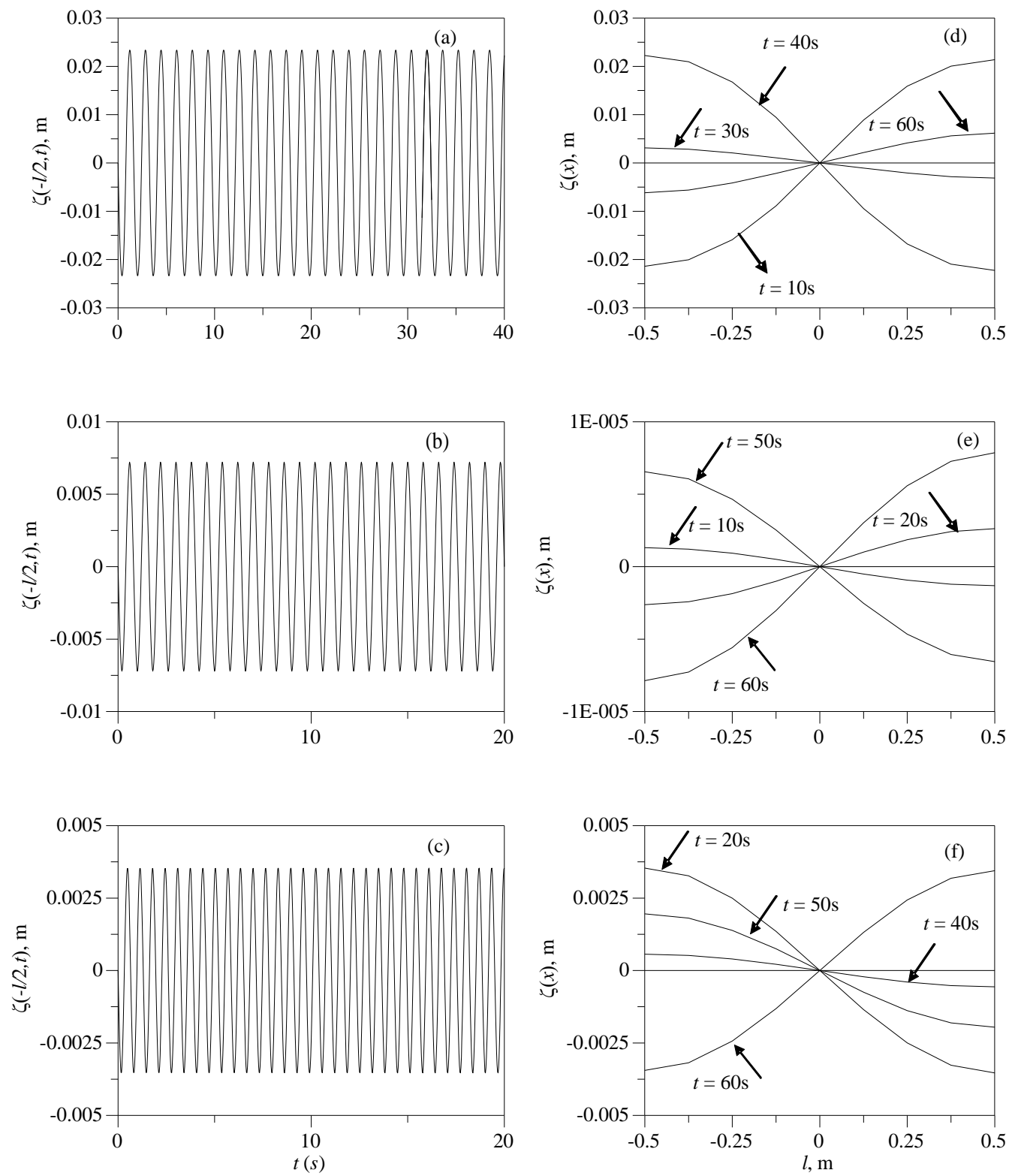

Fig. 17: Free surface profile along the length of the tank subjected to roll amplitude of $\phi_{\mathrm{o}}=0.1$ radians and excitation frequencies: (a) $f=0.79 f_{1}$, (b) $f=1.61 f_{1}$ and (c) $f=1.97 f_{1}$.

An attempt has been made to compare the trend (qualitative) in variation of sloshing oscillation due to coupled degree of excitation (sway, heave and roll) with the theoretical results of single (sway) degree of excitation. The experimental results for the aspect ratio $\left(h_{\mathrm{s}} / l\right)$ of 0.163 and 0.325 are shown in Fig. 20 and Fig. 21, respectively. Faltinsen (2000) theory has been exploited for the comparison purposes. It can be learnt that the experimental results of sloshing response follow the trends of theoretical work. It is also satisfy the nonlinear spring characteristics i.e the maximum response is observed to be away from classical resonance condition $\left(f_{w} / f_{1}=1\right)$. A secondary peak is seen at second lowest odd mode frequency $\left(f_{3}\right)$ due to resonance of third mode in the 
combined degree of excitation. In addition, it is learnt that the significance of individual degree of excitation is evident and must be understood prior to analyzing the results of combined degree of excitation.
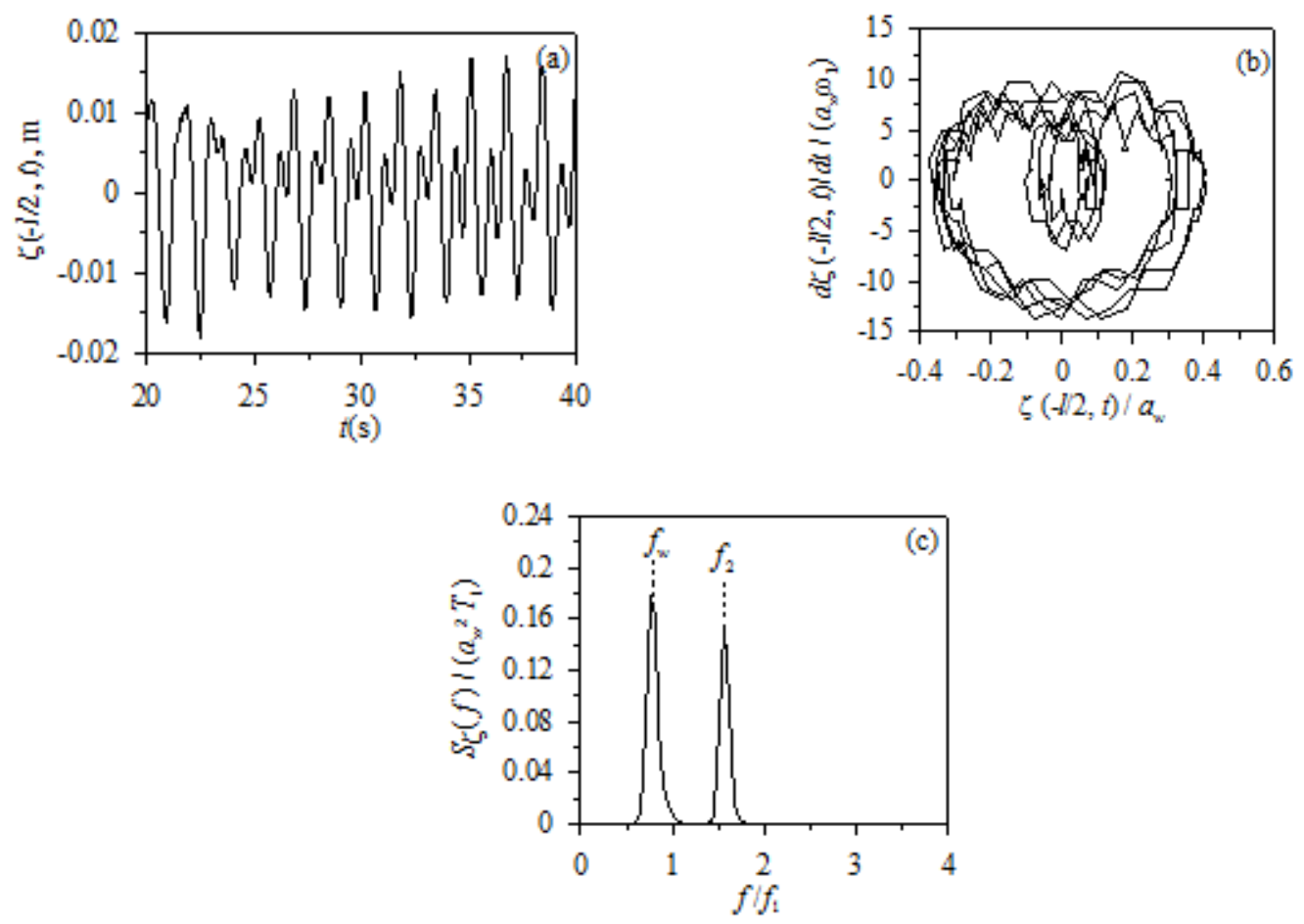

Fig. 18: Sloshing oscillation (Nasar et al. 2008a) for $h_{s} l l=0.325, H_{i} / d=0.08$ and $a_{w}=H_{i} / 2$ : (a) Surface elevation at the end wall, $f_{w} / f_{1}=0.80, X / H_{i}=0.70, Z / H_{i}=0.80$ and $\phi B / 2 H_{i}=1.01$; (b) phase plot and (c) surface elevation spectrum corresponding to (a).

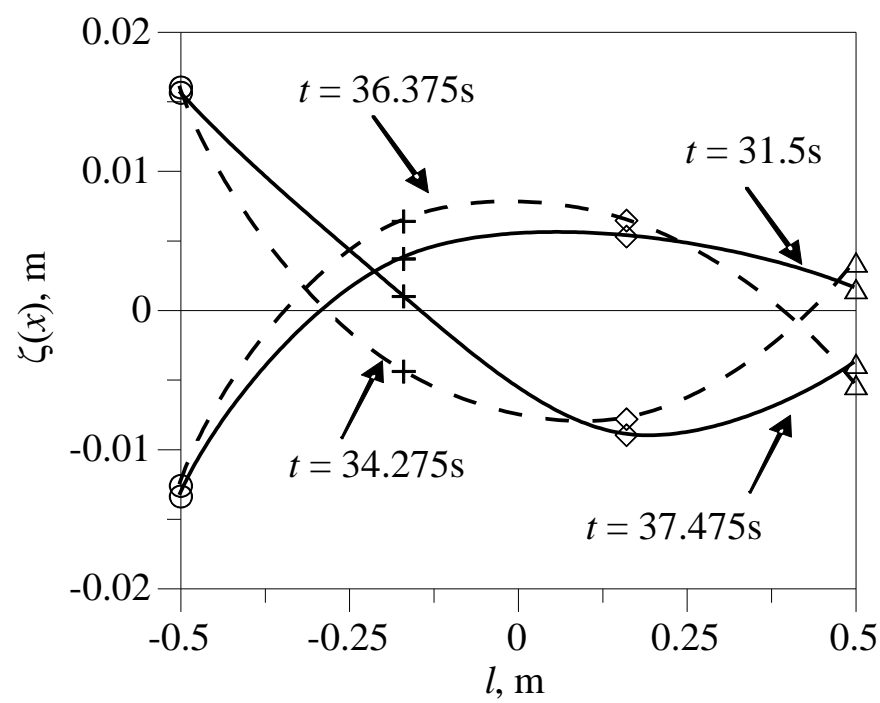

Fig. 19: Free surface profile (Nasar et al. 2008a) along the length of the tank for $h_{s} / l=0.325$ subjected to wave excitation frequency, $f_{w}=0.62 \mathrm{~Hz}$ and incident wave height, $H_{i}=0.08 \mathrm{~m}$. 


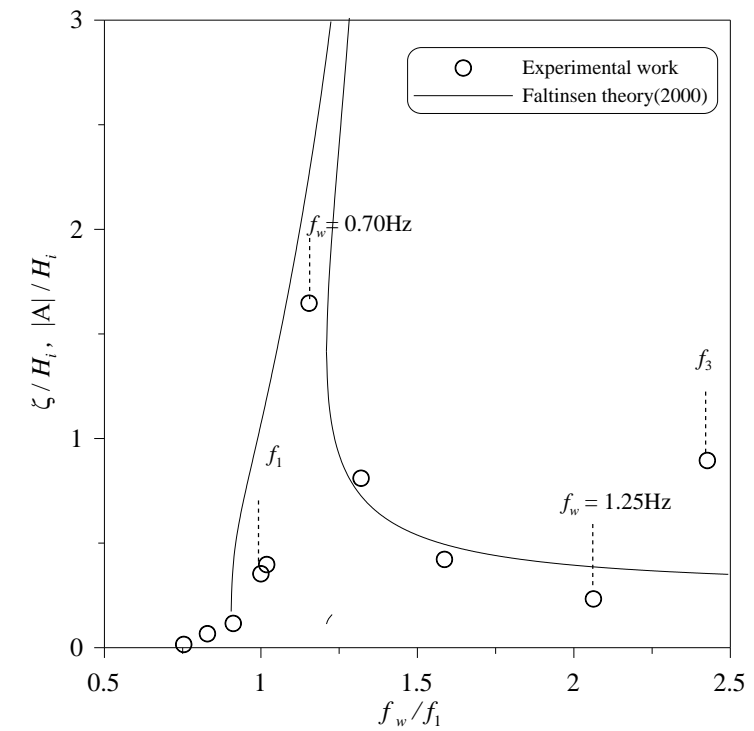

Fig. 20: Qualitative comparison of trend in variation of sloshing oscillation between experimental results (Nasar et al. 2008a) of coupled degree (sway, heave and roll) of excitation and theoretical results of single degree (sway) of excitation for an aspect ratio of $h_{s} / l=0.163$

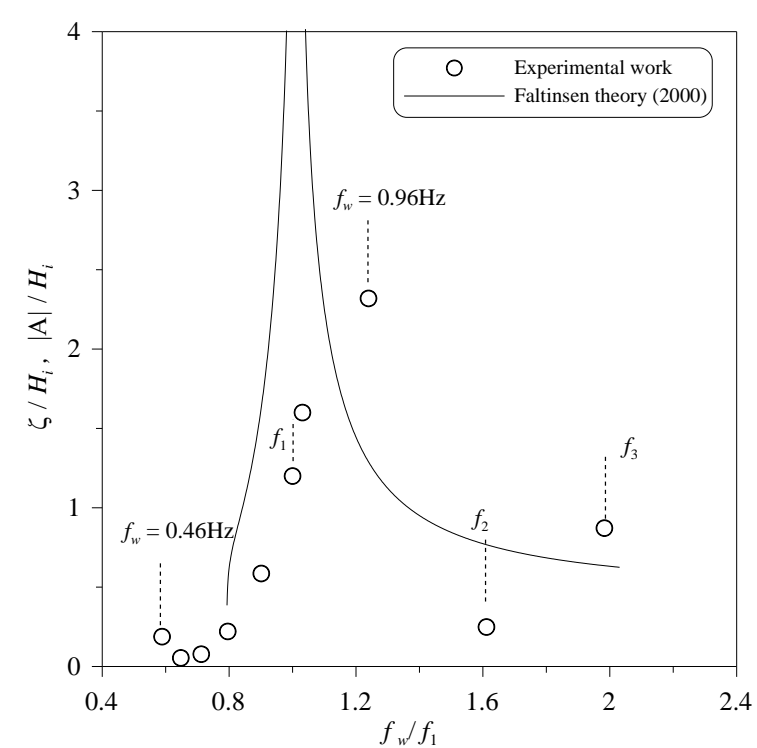

Fig. 21: Qualitative comparison of trend in variation of sloshing oscillation between experimental results (Nasar et al. 2008a) of coupled degree (sway, heave and roll) of excitation and theoretical results of single degree (sway) of excitation for an aspect ratio of $h_{s} / l=0.325$

\subsection{Conclusions}

In addition to the above discussion, based on the existing literature and authors' experience, the following general comments were made by considering the importance of degree of excitation. A partially filled tank in a ship experiences six degrees of excitation in the real marine environment. As a result, the induced sloshing oscillation and the resulting pressure on the liquid containment depends upon wave direction in which it approaches the vessel, the tank position in the vessel, wave frequency and shape of the tank (rectangular, circular or square). The liquid sloshing dynamics in a square tank subjected to excitation in yaw mode is quite important for design purpose which would be positioned in the fore and aft of the vessel. The mass of liquid movement is restricted to unidirectional while the tank is being subjected to either sway or roll excitation. Hence the sloshing pressure anticipated on the liquid containment is high. In general, the sway or surge (depends on the tank longitudinal axis with excitation direction) contributes high to their part for sloshing while comparing to the other degrees of excitation. The roll or yaw excitation comes to the next. The pitching effect is important in a case that the tank longitudinal axis positioned along the vessel's length. However, the heave excitation doesn't have any influence on disturbing the free surface unless it is combined with any other degrees of excitation. By keeping the above issues in mind and considering the importance of beam sea excitation, the present study explored the physics implicated with the sloshing phenomenon subjected to individual sway, heave and roll excitations. The following conclusions were drawn by solving the theoretical models.

Sway excited tank

- In general, the sloshing energy contribution by odd modes dominate even modes which means that odd modes are likely to be excited in sway excitation

- While $f_{w}<f_{1}$, the sloshing energy is observed at $f_{w}$ (dominant) and $f_{1}$. As the excitation frequency $\left(f_{w}\right)$ increases, energy concentrates at $f_{1}$ and diminishes at $f_{w}$. At resonance $\left(f_{w}=f_{1}\right)$, a single energy peak is observed at $f_{1}$.

- At $f_{w}>f_{1}$, energy starts to concentrate on higher order sloshing frequencies

Heave excited tank

- It is learnt that the static free surface of liquid would be remain undisturbed, unless, a particular mode shape $(n=1,2,3 \ldots \ldots)$ is assumed as perturbation in the heave excitation. It excites the same mode and the sloshing energy is amplified at parametric resonance condition $\left(f_{w}=2 f_{n}\right.$, $n=1,2,3 \ldots \ldots)$. 
Roll excited tank

- The sloshing energy is observed to be concentrate on excitation frequencies $\left(f_{w}\right)$ due to first mode contribution and at classical resonance $\left(f_{w}=f_{1}\right)$ the amplitude of sloshing oscillation is high.

The obtained knowledge is quite supportive in analyzing the sloshing dynamics (identification of harmonics and resonances) of combined degree of excitation of sway, heave and roll (authors' experimental work, Nasar et al.2008a). The existence of harmonics and mode shape was further confirmed with phase portrait and surface profile, respectively. Faltinsen (2000) modal theory has been exploited to obtain the nonlinear amplitudefrequency response curves for the aspect ratio $\left(h_{s} / l\right) 0.163,0.325,0.488$ and 0.585 considered in the experimental work. A qualitative comparison shows that the sloshing responses follow the trends of theoretical work and exhibit nonlinear characteristics.

\section{References}

Armenio, V. and La Rocca, M. (1996): On the Analysis of Sloshing of water in Rectangular Containers: Numerical study and Experimental validation, Ocean Engineering, Vol.23, No.3, pp.705-739. http://dx.doi.org/10.1016/0029-8018(96)84409-X

Benjamin, T.B. and Ursell, H.W. (1954): The Stability of the Plane Free Surface of a Liquid in a Vertical Periodic Motion, Proceedings of Royal Society of London, Series A225, pp.505 - 515. http://dx.doi.org/10.1098/rspa.1954.0218

British Maritime Corporate profile (2005): British Maritime Technology Limited, UK.

Faltinsen, O.M. (1974): Nonlinear Theory of Sloshing in Rectangular Tanks, Journal of Ship Research, Vol.18, No.4, pp. $224-241$.

Faltinsen, O.M. (1978): A Numerical Nonlinear Method of Sloshing in Tanks with Two Dimensional Flow, Journal of Ship Research, Vol.22, No.3, pp.193 - 202.

Faltinsen, O.M., Rognebakke, O.F., Lukovksky, I.A. and Timokha, A.N. (2000): Multimodal Analysis of Nonlinear Sloshing in a Rectangular Tank with Finite Water depth, Journal of Fluid Mechanics, Vol.407, pp.201 - 234. http://dx.doi.org/10.1017/S0022112099007569

Faltinsen, O.M. and Timokha, A.N. (2001): Adaptive Multimodal approach to Nonlinear Sloshing in a Rectangular Tank., Journal of Fluid Mechanics, Vol.432, pp.167 - 200.

Faltinsen, O.M. and Timokha, A.N. (2002): Asymptotic Modal approximation of Nonlinear Resonant Sloshing in a Rectangular Tank with small Fuid depth, Journal of Fluid Mechanics, Vol.470, pp.319 - 357. http://dx.doi.org/10.1017/S0022112002002112

Frandsen, J.B. (2003): Simulation of Sloshing motions in Fixed and Vertically Excited Containers using a 2-D Inviscid sigma-transformed Finite Difference Solver, Journal of Fluids and Structures, Vol.18, pp.197 - 214. http://dx.doi.org/10.1016/j.jfluidstructs.2003.07.004

Frandsen, J.B. (2004): Sloshing in Excited Tanks, Journal of Computational Physics, Vol.196, pp.53 - 87. http://dx.doi.org/10.1016/j.jcp.2003.10.031

Ibrahim, R.A. (2005): Liquid Sloshing Dynamics - Theory and Applications, Cambridge University press, Newyork.

Jermie, J.S., Sannasiraj S.A. and Sundar V. (2012): Numerical Simulation of Sloshing in a Rectangular Tank Subjected to Rotational Motion, Proceedings of the Twenty-second International Offshore and Polar Engineering Conference, Rhodes, Greece, June 17-22.

Jiang, L., Ting, C., Perlin, M. and Schultz, W.W. (1996): Moderate and steep Faraday waves: instabilities, modulation and temporal asymmetries, Journal of Fluid Mechanics, Vol.329, pp.275-307. http://dx.doi.org/10.1017/S0022112096008920

Lui, A.P. and Lou, Y.K. (1990): Dynamic Coupling of a Liquid Tank system under Transient Excitations, Ocean Engineering, Vol.17, No.3, pp.263 - 277. http://dx.doi.org/10.1016/0029-8018(90)90005-Q

Miles, J. and Henderson, D. (1990): Parametrically forced surface waves, Annual review of Fluid Mechanics, Vol. 22, pp.143-165. http://dx.doi.org/10.1146/annurev.fl.22.010190.001043

Moiseyev, N.N. (1958): On the Theory of Nonlinear Vibrations of a Liquid of Finite volume. Applied Mathematics and Mechanics, Vol. 22, No.5, pp.612 - 621.

Nasar T., Sannasiraj S.A. and Sundar V. (2008a): Experimental Study of Liquid Sloshing Dynamics in a Barge carrying Tank. Fluid Dynamics Research, $\quad$ Vol.40, pp.427 - 458. http://dx.doi.org/10.1016/j.fluiddyn.2008.02.001 
Nasar, T., Sannasiraj, S.A. and Sundar, V. (2008b): Sloshing Pressure Variation in a Barge carrying Tank, Ships and Offshore Structures, Vol.3, No.3, pp.185 - 203. http://dx.doi.org/10.1080/17445300802204363

Nasar, T., Sannasiraj, S.A. and Sundar, V. (2009): Wave Induced Sloshing pressure in a Liquid Tank under Irregular Waves, Part M: Journal of Engineering for Maritime Environment, Vol.223, No.2, pp.145 - 161. http://dx.doi.org/10.1243/14750902JEME135

Nasar, T., Sannasiraj, S.A. and Sundar, V. (2010): Motion Responses of Barge carrying Liquid Tank, Ocean Engineering, Vol.37, pp.935 - 946. http://dx.doi.org/10.1016/j.oceaneng.2010.03.006

Nasar, T., Sannasiraj, S.A. and Sundar, V. (2012): Liquid Sloshing Dynamics in a Barge subjected to Random Wave Excitations, Journal of Naval Architecture and Marine Engineering, Vol.9, No.1, pp.43-65. http://dx.doi.org/10.3329/jname.v9i1.7600

Ockendon, H., Ockendon, J.R and Johnson, A.D. (1989): Resonant Sloshing in Shallow water, Journal of Fluid Mechanics, Vol.167, pp.465-479. http://dx.doi.org/10.1017/S0022112086002926

Ockendon, H., Ockendon, J.R., Peake, M.R. and Chester, W.(1993): Geometrical effects in Resonance Gas Oscillations, Journal of Fluid Mechanics, Vol. $\quad 257, \quad$ pp.201 $\quad-\quad 217$. http://dx.doi.org/10.1017/S0022112093003040

Ockendon, H., Ockendon, J.R. and Waterhouse, D.D. (1996): Multimode Resonances in Fluids, Journal of Fluid Mechanics, Vol.315, pp.317 - 344. http://dx.doi.org/10.1017/S0022112096002443

Pirker, S., Aigner, A. and Wimmer, G. (2012): Experimental and Numerical investigation of Sloshing Resonance phenomena in a spring - mounted Rectangular Tank, Chemical Engineering Science, Vol.68, pp.143 - 150. http://dx.doi.org/10.1016/j.ces.2011.09.021

Solass, F. and Faltinsen, O.M.(1997): Combined Numerical and Analytical solution for Sloshing in TwoDimensional Tanks of General Shape, Journal of Ship Research, Vol.41, No.2, pp.118-129.

Sriram V., Sannasiraj S.A. and Sundar V. (2006): Numerical Simulation of 2D Sloshing Waves due to Horizontal and Vertical Random Excitations, Applied Ocean Research, Vol.28, pp.19 - 32. http://dx.doi.org/10.1016/j.apor.2006.01.002

The Naval Architect (September 2007): Simulating safe Shipping of LNG, The Royal Institution of Naval Architect, London, UK.

Warnitchai, P. and Pinkaew, T. (1998): Modeling of Liquid Sloshing in Rectangular Tanks with FlowDampening Devices, Engineering Structures, Vol.20, pp.593 - 600. http://dx.doi.org/10.1016/S0141$\underline{\text { 0296(97)00068-0 }}$

Waterhouse, D.D. (1994): Resonant Sloshing near Critical Depth, Journal of Fluid Mechanics, Vol.281, pp.313 - 318. http://dx.doi.org/10.1017/S0022112094003125

\section{Appendix -A}

Faltinsen et al. (2000) developed a general analytical model describing the free surface motion and velocity potential in generalized Fourier series. The variational principle is employed to derive the general modal system. For a rectangular tank of width, $l$ and liquid depth, $h_{s}$, the surface elevation is expressed as by the following series

$$
\zeta(x, t)=\sum_{n=1}^{\infty} q_{n}(t) f_{n}(x)
$$

where $q_{n}$ stand for the generalized coordinates and $f_{n}(x)$ are the mode shapes. The analysis of Faltinsen $e t$ al. (2000) yields the following systems of ordinary differential equations describing the modal oscillations of the liquid free surface under sway/surge motion $X^{\prime}(t)=-\omega_{h} a_{h} \sin \left(\omega_{h} t\right)$

$$
\begin{aligned}
& \ddot{q}_{1}+\omega_{1}^{2} q_{1}+c_{1}\left(\ddot{q_{1}} q_{2}+\dot{q}_{1} \dot{q}_{2}\right)+c_{2}\left(\ddot{q_{1}} q_{1}^{2}+\dot{q}_{1} q_{1}\right)+c_{3} \ddot{q}_{2} q_{1}+P_{1} X^{\prime}(t)=0 \\
& \ddot{q_{2}}+\omega_{2}^{2} q_{2}+c_{4} \ddot{q_{1}} q_{2}+c_{5} \dot{q}_{1}=0 \\
& \ddot{q}_{3}+\omega_{3}^{2} q_{3}+c_{6} \ddot{q}_{1} q_{2}+c_{7} \ddot{q}_{1} q_{1}^{2}+c_{8} \ddot{q}_{2} q_{1}+c_{9} \dot{q}_{1} \dot{q}_{2}+c_{10} \dot{q}_{1} q_{1}+P_{3} X^{\prime}(t)=0
\end{aligned}
$$


Equations (A.2), (A.3) and (A.4) are coupled through nonlinear inertia terms. By applying asymptotic approximation technique and assuming the solution of the first mode as $q_{1}(t)=A \cos \omega_{h}(t)+O(A)$, the steady state sloshing amplitude can be obtained by the following Equation (A.5)

$$
\left(r_{1}^{2}-1\right) \bar{A}+v\left(r_{2}, \bar{h}\right) \bar{A}^{3}-\overline{P_{1}} \bar{X}=0
$$

where

$$
\begin{aligned}
& v\left(r_{2}, \bar{h}\right)=\overline{c_{1}}\left(-\frac{\overline{c_{4}}-\overline{c_{5}}}{2 r_{2}^{2}}+\frac{\overline{c_{4}}+\overline{c_{5}}}{4\left(r_{2}^{2}-4\right)}\right)-\frac{1}{2} \overline{c_{2}}-\overline{c_{3}} \frac{\overline{c_{4}}+\overline{c_{5}}}{\left(r_{2}^{2}-4\right)} \\
& P_{2 n-1}=-\frac{4}{\pi(2 n-1)} \tanh \left((2 n-1) \pi h_{s} / l\right), c_{1}=\frac{\pi}{2 l}\left[\frac{1}{\tanh \left(\frac{\pi h_{s}}{l}\right)}+\tanh \left(\frac{\pi h_{s}}{l}\right)\right] \\
& c_{2}=\left(\frac{\pi}{2 l}\right)^{2}\left[-1+\frac{2}{\tanh \left(\frac{\pi h_{s}}{l}\right) \tanh \left(\frac{2 \pi h_{s}}{l}\right)}\right], c_{3}=\frac{\pi}{2 l}\left[\tanh \left(\frac{\pi h_{s}}{l}\right)-\frac{1}{\tanh \left(\frac{2 \pi h_{s}}{l}\right)}\right] \\
& c_{4}=\frac{\pi}{l}\left[\tanh \left(\frac{2 \pi h_{s}}{l}\right)-\frac{1}{\tanh \left(\frac{\pi h_{s}}{l}\right)}\right], c_{5}=\frac{\pi}{2 l}\left\{\tanh \left(\frac{\pi h_{s}}{l}\right)\left[1-\frac{1}{\left(\tanh \left(\frac{\pi h_{s}}{l}\right)\right)^{2}}\right]-\frac{2}{\tanh \left(\frac{\pi h_{s}}{l}\right)}\right\}
\end{aligned}
$$

The over - bar terms are nondimensional with respect to length $l$, and $r_{n}=\omega_{n} / \omega_{h}$, where $n=1,2,3$,

\section{Appendix -B}

Faltinsen (1974) developed a nonlinear theory to track the free surface oscillation based on the potential flow theory. A two-dimensional, rigid, rectangular, open tank without baffles is forced to oscillate harmonically with frequency $\omega$ and small amplitude of roll under steady state conditions. The free surface elevation inside the tank is given as follows

$$
\begin{aligned}
\zeta(x, t) & =\zeta_{1} \phi_{o}{ }^{1 / 3}+\zeta_{2} \phi_{o}{ }^{2 / 3}+\zeta_{3} \phi_{o} \\
\zeta_{1}(x, t) & =\frac{\omega_{1}^{2}}{\omega g} \sin \left(\frac{\pi x}{l}\right) \cosh \left(\frac{n \pi h_{s}}{l}\right) N \sin \omega t \\
\zeta_{2}(x, t) & =\frac{\omega_{1}^{2}}{\omega^{2} g} \cos 2 \omega t \cos \left(\frac{\pi x}{l / 2}\right) N^{2}\left(\frac{D-C}{4}+A_{1}^{(2)} \omega(\omega D-\omega C-g F) \cosh \left(\frac{\pi h_{s}}{l / 2}\right)\right) \\
& +\frac{\omega_{1}^{2}}{\omega^{2} g} \frac{N^{2}}{2}\left(\frac{D-E}{2}\right) \cos \left(\frac{\pi x}{l / 2}\right)
\end{aligned}
$$

For the sake of simplicity, the third order surface elevation $\left(\zeta_{3}\right)$ is not presented here due to its lengthy expression and involves several constants. However, the constants pertaining to the first and second order surface elevations are given as follows

$$
\begin{aligned}
& C=-\frac{\pi^{2}}{2 l^{2}} \sinh ^{2}\left(\frac{\pi h_{s}}{l}\right)-\frac{\omega_{1}^{2}}{g} \frac{\pi}{l} \cosh \left(\frac{\pi h_{s}}{l}\right) \sinh \left(\frac{\pi h_{s}}{l}\right), D=-\frac{\pi^{2}}{2 l^{2}} \cosh ^{2}\left(\frac{\pi h_{s}}{l}\right) \\
& F=\frac{\pi^{2}}{l^{2}} \frac{\omega}{g} \cosh ^{2}\left(\frac{\pi h_{s}}{l}\right), E=-\frac{\pi^{2}}{2 l^{2}} \sinh ^{2}\left(\frac{\pi h_{s}}{l}\right)+\frac{\omega_{1}^{2}}{g} \frac{\pi}{l} \cosh \left(\frac{\pi h_{s}}{l}\right) \sinh \left(\frac{\pi h_{s}}{l}\right)
\end{aligned}
$$

To avoid secular terms, we have to set

$\left(K_{1}-K_{2}\right) N^{3}+\alpha_{1} \cosh \left(\frac{\pi h_{s}}{l}\right) N+\omega^{3} \frac{8 a}{\pi^{2}}\left(-z_{1}-\frac{2 l \tanh \left(\frac{\pi h_{s}}{2 l}\right)}{\pi}+\frac{g}{\omega_{1}^{2}}\right)=0$ in Equations (B.2) and

(B.3). This determines N. In which, $\omega^{2}=\omega_{1}^{2}+\phi_{o}{ }^{2 / 3} \alpha_{1}$ 


$$
\begin{aligned}
& K_{1}=\frac{3 \pi^{2}}{2 l^{2}} \omega^{2} A_{1}^{(2)}\left(\frac{\pi^{2}}{4 l^{2}} \cosh \left(\frac{\pi h_{s}}{l}\right)-\frac{\omega_{1}^{2}}{g} \frac{\pi}{4 l} \sinh \left(\frac{\pi h_{s}}{l}\right) \cosh \left(\frac{\pi h_{s}}{l}\right)\right)-\frac{\omega_{1}^{2}}{g} \frac{5 \pi^{3}}{32 l^{3}} \sinh \left(\frac{\pi h_{s}}{l}\right) \\
& +\frac{\omega_{1}^{2}}{g} \frac{7 \pi^{3}}{32 l^{3}} \sinh \left(\frac{\pi h_{s}}{l}\right) \cosh ^{2}\left(\frac{\pi h_{s}}{l}\right) \\
& K_{2}=-A_{1}^{(2)} \frac{3 \omega^{2} \pi^{4}}{4 l^{4}} \cosh \left(\frac{\pi h_{s}}{l}\right) \cos \left(\frac{2 \pi h_{s}}{l}\right)-\frac{3 \pi^{4}}{32 l^{4}} \cosh \left(\frac{\pi h_{s}}{l}\right) \\
& -\frac{\omega_{1}^{2}}{g} \frac{\pi^{3}}{32 l^{3}} \sinh \left(\frac{\pi h_{s}}{l}\right) \cosh ^{2}\left(\frac{\pi h_{s}}{l}\right) \\
& A_{1}^{(2)}=\left[-4 \omega^{2} \cosh \left(\frac{2 \pi h_{s}}{l}\right)+\frac{\omega^{2}}{\omega_{1}^{2}} g \frac{2 \pi}{l} \sinh \left(\frac{2 \pi h_{s}}{l}\right)\right]^{-1} \\
& \text { if } 4 \omega_{1}^{2} \neq g \frac{2 \pi}{l} \tanh \left(\frac{2 \pi h_{s}}{l}\right)
\end{aligned}
$$

